\title{
Alexithymia, suicidal ideation, and serum lipid levels among drug-naïve outpatients with obsessive-compulsive disorder
}

\author{
Domenico De Berardis, ${ }^{1,2}$ Nicola Serroni, ${ }^{1}$ Stefano Marini, ${ }^{1}$ Gabriella Rapini, ${ }^{1}$ Alessandro Carano, ${ }^{2}$ \\ Alessandro Valchera, ${ }^{3}$ Felice lasevoli, ${ }^{4}$ Monica Mazza, ${ }^{5}$ Maria Signorelli, ${ }^{6}$ Eugenio Aguglia, ${ }^{6}$ \\ Giampaolo Perna, ${ }^{7,8,9}$ Giovanni Martinotti, ${ }^{3}$ Paola A. Varasano, ${ }^{10}$ Gabriella Lucidi Pressanti, ${ }^{10}$ \\ Massimo Di Giannantonio ${ }^{2}$
}

\begin{abstract}
${ }^{1}$ Psychiatric Service of Diagnosis and Treatment, Department of Mental Health, G. Mazzini Hospital, ASL 4, Teramo, Italy. ${ }^{2}$ Department of Neurosciences and Imaging, Chair of Psychiatry, Università degli Studi G. d'Annunzio Chieti e Pescara, Chieti Scalo, Italy. ${ }^{3}$ Casa di Cura Villa San Giuseppe, Ascoli Piceno, Italy. ${ }^{4}$ Section of Psychiatry, Department of Neuroscience, School of Medicine, Università degli Studi di Napoli Federico II, Naples, Italy. ${ }^{5}$ Department of Science of Health, Università degli Studi dell'Aquila, L'Aquila, Italy. ${ }^{6}$ Department of Clinical and Molecular Biomedicine, Università degli Studi di Catania, Catania, Italy. ${ }^{7}$ Department of Psychiatry and Neuropsychology, University of Maastricht, Maastricht, The Netherlands. ${ }^{8}$ Department of Clinical Neurosciences, Villa San Benedetto Menni, Albese con Cassano, Como, Italy. ${ }^{9}$ Department of Psychiatry and Behavioral Sciences, Leonard M. Miller School of Medicine, University of Miami, Florida, USA.

${ }^{10}$ Department of Immunohematology and Transfusional Medicine, G. Mazzini Hospital, ASL 4, Teramo, Italy.
\end{abstract}

\begin{abstract}
Objective: As obsessive-compulsive disorder (OCD) is a relatively common psychiatric disorder with a significant suicide risk, the individuation of potential biomarkers of suicidality, such as cholesterol levels, may enable recognition of at-risk subjects. Therefore, the aims of this study were to: 1) evaluate potential differences in clinical and laboratory parameters between patients with and without alexithymia and compare them with healthy controls; and 2) investigate which clinical and laboratory variables were associated with suicidal ideation.

Methods: 79 drug-naïve adult outpatients with a DSM-IV diagnosis of OCD were recruited. Alexithymia was measured with the 20-item Toronto Alexithymia Scale (TAS-20), suicidal ideation was assessed with the Scale for Suicide Ideation, and depressive symptoms were evaluated with the Montgomery-Åsberg Depression Rating Scale (MADRS). Serum lipid levels of 40 healthy controls were also evaluated.

Results: Alexithymic patients had altered serum lipid levels in comparison with non-alexithymics and healthy controls. Using a linear regression model, the presence of symmetry/ordering obsessions and compulsions, lower HDL-C levels, and difficulty in identifying feelings dimension of the TAS-20 were associated with higher suicidal ideation.

Conclusions: Alexithymic individuals with OCD may exhibit dysregulation of the cholesterol balance, which in turn may be linked to suicidal ideation. Further prospective studies are required to elucidate this potential association.
\end{abstract}

Keywords: Alexithymia; obsessive-compulsive disorder; suicide ideation; serum lipid levels; drugnaïve

\section{Introduction}

The alexithymia construct, formulated from clinical investigations, is multifaceted and includes four different characteristics: a) difficulty in identifying and describing feelings; b) difficulty in distinguishing feelings from bodily sensations; c) a reduction in fantasy; and d) concrete and minimally introspective thinking. ${ }^{1}$ Alexithymic individuals also suffer from affective dysregulation, the inability to

Correspondence: Domenico De Berardis, Department of Mental Health, Psychiatric Service of Diagnosis and Treatment, G. Mazzini Hospital, p.zza Italia 164100 , Teramo, Italy.

E-mail: dodebera@aliceposta.it

Submitted Jun 21 2013, accepted Aug 172013. self-soothe and manage emotions because of a lack of emotional awareness. ${ }^{1}$ Alexithymia may be present in 30 to $40 \%$ of patients with obsessive-compulsive disorder $(\mathrm{OCD})^{2}$ and has also been linked to increased severity and lower insight. ${ }^{3}$ Contrary to common belief, OCD patients may be at risk of committing suicide, ${ }^{4}$ and the presence of alexithymia may further increase this risk. ${ }^{5}$ In fact, several studies have showed that alexithymic features may be positively associated with a history of attempted suicide and increased suicide risk even in absence of depressive symptoms. ${ }^{6}$ Undoubtedly, as suggested by Pompili, $^{7}$ individuals with unbearable psychological pain sometimes cannot find real words to express the state they are experiencing. Therefore, 
alexithymic subjects may be more prone to express their unbearable psychological pain as a suicidal act. ${ }^{8}$

The role of serum lipid levels in neuropsychiatric disorders has been widely investigated. ${ }^{9,10}$ Some data suggest that the serum lipid profile may be altered in patients with OCD as compared with normal controls, ${ }^{11,12}$ but, to date, findings have been somewhat inconsistent. Moreover, it has been reported that serum lipid levels may be related to suicidal ideation and external-cause mortality, ${ }^{13,14}$ and this may be particularly true for low serum cholesterol levels. ${ }^{15}$

As OCD is a relatively common psychiatric disorder that carries a significant suicide risk, the individuation of potential biomarkers of suicidality, such as cholesterol levels, may permit the recognition of at-risk subjects. ${ }^{4}$ However, to date, the interrelationships between alexithymia, suicidal ideation, and serum lipid profile in OCD patients have not been studied. Furthermore, evaluating OCD patients with a relatively long history of illness may lead to potential biases because of current or previous pharmacological treatments that may themselves influence alexithymia and serum lipid levels. Therefore, in the present exploratory study, we evaluated only drug-naïve patients with OCD. Specifically, our aims were to: 1) evaluate potential differences in clinical and laboratory parameters between patients with and without alexithymia and compare them to healthy controls; and 2) investigate which clinical and laboratory variables were associated with suicidal ideation using a blockwise linear regression analysis.

\section{Methods}

\section{Subjects}

Patients aged 18 to 44 years with a diagnosis of OCD at first episode who had never received pharmacological or psychotherapeutic treatment were considered eligible for this study. Diagnoses were made by clinical assessment following the Structured Clinical Interviews for DSM-IV Axis I Disorders (SCID-I). The participants enrolled in the study had a total Yale-Brown Obsessive Compulsive Scale $\left(\mathrm{Y}\right.$-BOCS) ${ }^{16}$ score of at least 16 within the first 10 items.

Exclusion criteria included: any concomitant axis I disorder; organic mental disorders; mental retardation; pregnancy or nursing; known familial hypercholesterolemia; and obesity. Subjects with diabetes, other endocrine disorders, hypertension, liver dysfunction, or other conditions necessitating any chronic pharmacotherapy were excluded. Patients who received medication for cholesterol dysregulation in the last 6 months and those whose body mass changed substantially within the 4 weeks prior to screening were also excluded from the study. Subjects with an $\mathrm{IQ} \leqslant 70$ as measured by the Wechsler Adult Intelligence Scale-Revised (WAIS-R) were excluded from the study to avoid potential biases in interpreting results of the Toronto Alexithymia Scale (TAS-20). Past or current substance abuse was also considered as an exclusion criterion.
From January 2010 to December 2010, we recruited 90 consecutive drug-naïve adult outpatients with a diagnosis of OCD at the Mental Health Center of University of Chieti. Of the 90 recruited patients, $10(11.1 \%)$ met exclusion criteria and one $(1.1 \%)$ refused to participate. Therefore, a total of 79 drug-naïve OCD outpatients were included in the analysis ( $87.8 \%$ participation rate).

\section{Procedures}

The rating scales were administered by psychiatrists with at least 5 years' clinical experience, who were supervised by senior psychiatrists (NS, EA, MDG).

Severity of OCD was assessed with the first 10 items of the Y-BOCS. The clinician-administered version of the YBOCS symptom checklist was used to assess the presence of five symptom dimensions, designated as aggression/checking, contamination/cleaning, hoarding, sexual/religious obsessions, and symmetry/ordering, as suggested by Mataix-Cols et al. ${ }^{17}$ In our sample, the interrater reliability for the Y-BOCS total score was $0.93(p<$ 0.001).

Alexithymia was evaluated using the Italian version of the 20-item TAS-20. ${ }^{18}$ A score of 61 or higher was considered indicative of alexithymia. The TAS-20 has a three-factor structure: Factor I assesses capacity to identify feelings and to distinguish between feelings and bodily sensations of emotional arousal (difficulty in identifying feelings [DIF]); Factor 2 reflects inability to communicate feelings to other people (difficulty in describing feelings [DDF]); and Factor 3 assesses externally-oriented thinking (EOT). The mean TAS-20 total score was $52.2 \pm 13.7 ; 30.4 \%(n=24)$ of 79 patients who scored 61 or more were considered positive for alexithymia. In our sample, Cronbach's alpha for the TAS-20 scale was 0.87 .

The Beck Scale for Suicide Ideation (SSI), ${ }^{19}$ a 3-point clinician-rated scale with statements of suicidal intention, was used to assess suicidal ideation. In our sample, the inter-rater reliability for this instrument was 0.89 ( $p<$ $0.001)$.

The Montgomery-Åsberg Depression Rating Scale (MADRS) was used to evaluate depressive symptoms. ${ }^{20}$ In our sample, the inter-rater reliability was 0.95 ( $p<$ 0.001 ) and the mean score was $7.1 \pm 2.0$.

Weight was measured (with patients barefoot and wearing light indoor clothing) using a balance beam scale, and height was measured using a stadiometer. Weight and height were used to calculate body mass index (BMI), expressed as $\mathrm{kg} / \mathrm{m}^{2}$.

The following parameters were measured in all subjects: high-density lipoprotein cholesterol (HDL-C), lowdensity lipoprotein cholesterol (LDL-C), total cholesterol (TC), triglyceridemia (TG), and very-low-density lipoprotein cholesterol (VLDL-C). The TC/HDL-C and LDL-C/ HDL-C ratios were also evaluated. Serum lipid concentrations were determined by enzymatic methods with an Abbott ABA-100 Bichromatic analyzer (Abbott, U.S.). Cholesterol and serum triglycerides were also measured using enzymatic methods (cholesterol: Boehringer 
Mannheim, Germany; serum triglycerides: Abbott). Serum HDL cholesterol was measured after phosphotungstic acid/ $\mathrm{MgCl}_{2}$ precipitation. LDL-C and VLDL-C concentrations were estimated by the Friedewald formula. Blood samples were taken between 7:00 and 8:30 a.m. after the patients had fasted for at least 10 hours and after a psychiatric evaluation. The serum lipid profile of a control group of 40 normal subjects (20 males and 20 females), with a mean age of $28.3 \pm 6.9$ and a mean BMI of $22.2 \pm 1.8 \mathrm{~kg} / \mathrm{m}^{2}$, recruited from faculty and hospital staff, was also evaluated.

The study was approved by the ethics committee of the University of Chieti. Each patient and healthy control had to understand the nature of the study, and all signed an informed consent document prior to laboratory testing and psychiatric evaluation.

\section{Statistics}

Descriptive statistics and percentages were computed for all demographic variables and psychometric scales. All demographic, clinical, and laboratory variables in the present study were checked for deviations from the Gaussian distribution using the Kolmogorov-Smirnov test. Given the normal distribution of the variables, one-way analysis of variance (ANOVA) was used to analyze gender differences. Differences between individuals with and without alexithymia were tested using analyses of covariance (ANCOVA) with TAS-20 positivity/negativity as a factor and age, gender, BMI, and MADRS scores as covariates. Post-hoc analysis was done by employing Bonferroni tests. Effect size (Cohen's d) was calculated by dividing the difference between the mean scores of alexithymic and non-alexithymic patients by the pooled standard deviation (SD). Cohen describes d values of 0.2 as denoting a small effect size, 0.5 as medium, and 0.8 as a large effect. Blockwise linear regression analyses were performed to ascertain which variables were associated with suicidal ideation (SSI as dependent variable). BMI, age, gender, duration of illness, MADRS, Y-BOCS subscales, and the five Y-BOCS Symptom Checklist dimensions were added in the first block. In the second block, HDL-C, LDL-C, TC, TG, VLDL-C, TC/HDL-C, and LDL-C/HDL-C were added to the models. The DIF, DDF, and EOT subscales of the TAS-20 were entered in the last step of both models. The quality of the regression model was tested using the Durbin-Watson statistic. Variance inflation factor (VIF) values were used to detect potential multicollinearity. P-values $\leqslant 0.05$ were deemed statistically significant. All statistical tests were two-sided. Statistical analyses were performed with SPSS version 10.0.0 (2000).

\section{Results}

The sample consisted of 43 men and 36 women with a mean age of $28.7 \pm 8.0$ years, a mean duration of illness of $6.7 \pm 4.4$ years, and a mean BMl of $22.0 \pm 1.7 \mathrm{~kg} / \mathrm{m}^{2}$, with no differences between alexithymics and nonalexithymics. Five patients $(6.3 \%)$ had attempted suicide at some point in their life, whereas seven (8.9\%) reported a family history of suicide. Gender comparisons between all demographic and clinical variables showed no significant differences.

Comparison between individuals with or without alexithymia, controlling for age, gender, BMI, and MADRS scores, revealed that alexithymics had higher total $Y$ BOCS, Y-BOCS obsessive subscale, Y-BOCS compulsive subscale, and SSI scores than non-alexithymics (Table 1). Moreover, they exhibited higher TG and VLDL$C$ and lower TC, HDL-C, and LDL-C levels than nonalexithymics and healthy controls. Finally, TC/HDL-C and LDL-C/HDL-C ratios were higher in alexithymics as compared with non-alexithymics and healthy controls. Effect size calculation showed that the magnitude of the group effect between alexithymics and non-alexithymics concerning Y-BOCS and its subscales, SSI, TC, HDL-C, LDL-C, VLDL-C, TG, and TC/HDL-C was large, whereas the effect for LDL-C/HDL-C was small. Comparison of serum lipid levels revealed no differences between nonalexithymics and healthy controls.

In the linear regression model (Table 2), presence of symmetry/ordering obsessions and compulsions, lower HDL-C levels, and DIF dimension of TAS-20 were associated with greater suicidal ideation (SSI as dependent variable). In the current analyses, the $R^{2}$ values accounted for $65 \%$ of variance in SSI. In addition, the Durbin-Watson coefficient was 1.83 (near the optimal value of 2.0) and the VIF values for all statistically significant variables were below 2.5 , indicating absence of collinearity. A scatterplot of residuals and a plot of regression-standardized residuals indicated a near-normal distribution.

\section{Discussion}

To the best of our knowledge, this was the first study to evaluate the relationships between alexithymia, suicidal ideation, and serum lipid levels in a sample of drug-naïve adult outpatients with OCD.

Alexithymics had higher TG, VLDL-C, TC/HDL-C, and LDL-C/HDL-C and lower TC, HDL-C, and LDL-C levels than non-alexithymics and healthy controls, as well as increased OCD severity. These results may be explained by Freyberger's concept ${ }^{21}$ of acute "secondary alexithymia" as a reaction to stressful situations. Acute secondary alexithymia is described as a transient, statedependent phenomenon that results as a consequence of personal distress, and which may decrease once an acute disease episode has resolved. As alexithymics had higher Y-BOCS and SSI scores than non-alexithymics, serum lipid alterations may reflect a state-dependent phenomenon possibly linked to greater disorder severity.

In our study, alexithymics exhibited greater suicidal ideation than non-alexithymics, and this was associated with lower HDL-C levels as well as with the DIF dimension of TAS-20, regardless of the presence of depressive symptoms. It has been reported that patients with high suicidal ideation may have lower serum HDL-C concentrations, $^{22}$ which also seems to apply to our 
Table 1 Comparison of Y-BOCS, SSI, and serum lipid levels between individuals with alexithymia (patients with a TAS-20 score $\geqslant 61$ ), without alexithymia (patients with a TAS-20 score $\leqslant 61$ ), and healthy controls, controlling for age, gender, BMI, and MADRS scores (data expressed as mean $\pm \mathrm{SD}$ )

\begin{tabular}{|c|c|c|c|c|c|c|c|}
\hline & $\begin{array}{l}\text { Overall } \\
(n=79)\end{array}$ & $\begin{array}{l}\text { Scores } \geqslant 61 \\
\text { on TAS-20 } \\
(n=24, \\
30.4 \%) \\
(I)\end{array}$ & $\begin{array}{l}\text { Scores } \leqslant 61 \\
\text { on TAS-20 } \\
(n=55 \\
69.6 \%) \\
(I I)\end{array}$ & $\begin{array}{l}\text { Controls } \\
(\mathrm{n}=40) \\
(\mathrm{III})\end{array}$ & $\begin{array}{l}\text { Between groups } \\
\text { comparison } \\
(\text { ANCOVA) }(\mathrm{df}=1.78)\end{array}$ & $\begin{array}{l}\text { Post-hoc } \\
\text { analysis } \\
\text { (Bonferroni) } \\
\text { p-values }\end{array}$ & $\begin{array}{l}\text { Effect size } \\
\text { (Cohen's d), } \\
\text { alexithymics } \\
\text { vs. non- } \\
\text { alexithymics }\end{array}$ \\
\hline \multicolumn{8}{|l|}{ Y-BOCS } \\
\hline Total score & $26.0 \pm 5.4$ & $32.5 \pm 4.1$ & $23.2 \pm 2.9$ & - & $F=138.6, p<0.001$ & - & 2.61 \\
\hline Obsessive subscale & $14.1 \pm 2.8$ & $16.9 \pm 2.4$ & $12.8 \pm 1.9$ & - & $F=68.6, p<0.001$ & - & 1.54 \\
\hline $\begin{array}{l}\text { Compulsive } \\
\text { subscale }\end{array}$ & $11.9 \pm 3.1$ & $15.5 \pm 2.3$ & $10.3 \pm 1.7$ & - & $F=118.7, p<0.001$ & - & 2.57 \\
\hline SSI & $3.3 \pm 3.2$ & $7.2 \pm 2.9$ & $1.6 \pm 1.2$ & - & $F=143.8, p<0.001$ & - & 2.52 \\
\hline TC (mg/dL) & $183.4 \pm 16.6$ & $172.1 \pm 12.2$ & $188.4 \pm 12.9$ & $184.3 \pm 25.0$ & $F=29.3, p<0.001$ & $0.001 \mathrm{I}<\mathrm{II}, \mathrm{III}$ & 1.30 \\
\hline HDL-C (mg/dL) & $51.7 \pm 8.6$ & $44.5 \pm 3.4$ & $54.8 \pm 8.4$ & $53.6 \pm 9.4$ & $F=45.6, p<0.001$ & $0.001 \mathrm{I}<\mathrm{II}, \mathrm{III}$ & 1.61 \\
\hline LDL-C (mg/dL) & $106.6 \pm 14.8$ & $97.6 \pm 11.5$ & $110.6 \pm 14.4$ & $107.4 \pm 17.3$ & $F=16.1, p<0.001$ & $0.001 \mathrm{I}<\mathrm{II}, \mathrm{III}$ & 1.00 \\
\hline VLDL-C (mg/dL) & $23.7 \pm 5.5$ & $28.1 \pm 4.3$ & $21.8 \pm 4.8$ & $21.2 \pm 5.8$ & $F=81.2, p<0.001$ & $0.001 \mathrm{I}>\mathrm{II}, \mathrm{III}$ & 1.38 \\
\hline TG (mg/dL) & $127.7 \pm 18.5$ & $140.8 \pm 22.7$ & $122.0 \pm 12.9$ & $124.4 \pm 20.1$ & $F=21.2, p<0.001$ & $0.001 \mathrm{I}>\mathrm{II}, \mathrm{III}$ & 1.02 \\
\hline TC/HDL-C ratio & $3.6 \pm 0.5$ & $3.9 \pm 0.4$ & $3.5 \pm 0.5$ & $3.5 \pm 0.6$ & $F=14.9, p<0.001$ & $0.001 \mathrm{I}>\mathrm{II}, \mathrm{III}$ & 0.88 \\
\hline LDL-C/HDL-C ratio & $2.1 \pm 0.4$ & $2.2 \pm 0.3$ & $2.1 \pm 0.4$ & $2.1 \pm 0.5$ & $F=3.9, p=0.05$ & $0.05 \mathrm{I}>\mathrm{II}, \mathrm{III}$ & 0.28 \\
\hline
\end{tabular}

ANCOVA = analyses of covariance; $\mathrm{BMI}=$ body mass index; $\mathrm{df}=$ degrees of freedom; $\mathrm{HDL}-\mathrm{C}=$ high-density lipoprotein cholesterol; $\mathrm{LDL}-\mathrm{C}=$ low-density lipoprotein cholesterol; MADRS = Montgomery-Åsberg Depression Rating Scale; SD = standard deviation; SSI = Scale for Suicide Ideation; TAS-20 = Toronto Alexithymia Scale; TC = total cholesterol; TG = triglyceridemia; VLDL-C = very-low-density lipoprotein cholesterol; Y-BOCS = Yale-Brown Obsessive Compulsive Scale.

results. Several studies have focused on associations between alexithymia and suicidal behaviors and have found that alexithymia may be positively associated with increased suicide risk. ${ }^{23,24}$ Kauhanen et al. ${ }^{25}$ found that alexithymics had a threefold greater risk of death from accidents, injury, or violence, including suicide, when compared to non-alexithymics. In a prospective study, Hintikka et al. ${ }^{11}$ confirmed that suicidal ideation was more common among subjects with alexithymia than among non-alexithymics. Moreover, the finding of an association between the DIF dimension of TAS-20 and suicidal ideation is consistent with the results of a previous study $^{26}$ that demonstrated that the DIF dimension of the TAS-20 is often related to suicidal ideation, even in absence of depressive symptoms.

In fact, it bears noting that, in our sample, depressive symptoms were not associated with increased suicidal ideation. This finding is in accordance with the work of Torres et al., ${ }^{27}$ who reported that depressive symptoms were associated with previous suicidal ideation but not with current suicidal ideation or with suicide attempts. Apter et al. ${ }^{28}$ even found an inverse relationship between depression and suicidal behavior in a sample of adolescents with OCD. Clinicians should be aware that the absence of clinically relevant depressive symptoms does not rule out suicidal ideation, and should screen directly for suicidal ideation when indicated. Moreover, in our sample, symmetry/ordering obsessions and compulsions were significantly associated with suicidal ideation, which is consistent with a previous study. ${ }^{5}$

Taken together, the results of our study support the notion that alexithymic individuals with OCD may have a dysregulation of cholesterol balance, which, in turn, may be associated with suicidal ideation. The mechanism of this association is unclear. Decreased serum cholesterol levels may reduce cholesterol levels in the brain, which may lead to reduced synaptic plasticity and brain dysfunction associated with impaired neurobehavioral consequences. ${ }^{29}$ It has been reported that suicide completers using violent methods were found to have significantly lower cholesterol content in the frontal cerebral cortex as compared with non-violent suicide completers, particularly in the orbitofrontal cortex (OFC) and the ventral prefrontal cortex. ${ }^{30,31}$ There seems to be

Table 2 Results of linear regression analysis with SSI scores as dependent variable and other variables as independent (only statistically significant variables are shown)

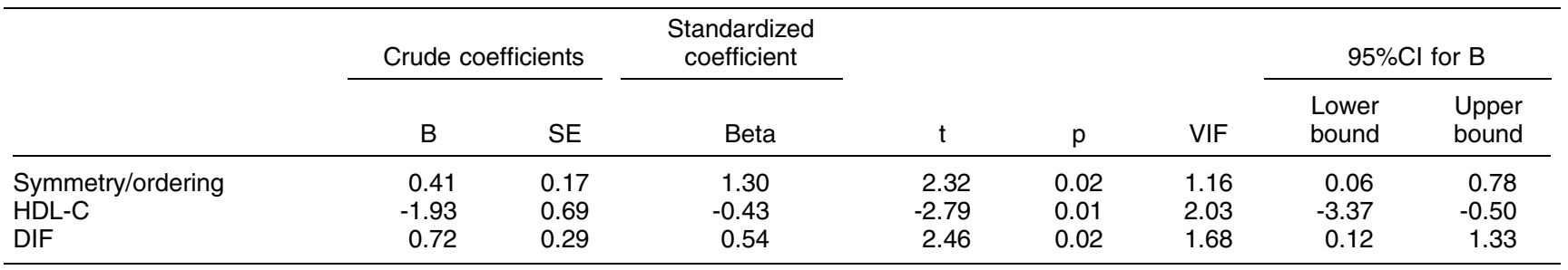

$95 \% \mathrm{Cl}=95 \%$ confidence interval; DIF = difficulty in identifying feelings; $\mathrm{HDL}-\mathrm{C}=$ high-density lipoprotein cholesterol; SE = standard error; $\mathrm{SSI}=$ Scale for Suicide Ideation; VIF = variance inflation factor.

$R^{2}=0.65 ; \mathrm{F}=10.04 ; \mathrm{df}=19 ; \mathrm{p}<0.001$. 
a connection between vascular serotonergic sensitivity and serum cholesterol levels. ${ }^{14}$ Vevera et al. ${ }^{32}$ showed that pharmacological cholesterol-lowering therapy altered serotonergic transmission. Low peripheral and central cholesterol levels can reduce the lipid viscosity of neuronal cell membranes, which may decrease exposure of pre-synaptic serotonin transporter or post-synaptic serotonin receptors, ${ }^{33}$ leading to decreased serotonin activity, which may in turn increase tendencies toward impulsive, aggressive, and suicidal behavior. ${ }^{34}$ Cholesterol deficiency in neuronal membranes may also have a direct negative impact on synapse formation, dendritic outgrowth, and neuronal survival. ${ }^{35}$ Moreover, as several studies have ${ }^{36,37}$ shown that OCD patients may have increased oxidative stress and that this correlates with disease severity, we may speculate that lipid peroxidation might be one of the causes of reduced serum HDL-C and altered lipid profile in these patients.

Alexithymia probably results from an inhibitory mechanism that could contribute to a suppression of emotional processing within the right hemisphere and to poorer verbalization of emotional contents (within the left hemisphere). ${ }^{38}$ Kano et al., ${ }^{39}$ using positron emission tomography (PET), showed that alexithymic males had lower regional cerebral blood flow in the inferior and middle frontal cortex, OFC, inferior parietal cortex, and occipital cortex in the right hemisphere than nonalexithymics while viewing a range of emotional facial expressions. These findings were also largely confirmed by Berthoz et al. ${ }^{40}$ in a functional magnetic resonance imaging (fMRI) study. As dyslipidemia (in particular, low TC and HDL-C levels) may be associated with outwardly directed aggression and self-harm behaviors in patients with various diagnoses, this may be the result of a hypothalamic-pituitary-adrenal (HPA) axis disorder caused by stress and neurobiological changes in central lipids, particularly in the OFC and ventral parietal cortex (VPC). ${ }^{41}$ Therefore, OFC and VPC dysfunction in alexithymics may cause a perturbation of HPA functioning, leading to a dyslipidemia that may be associated with increased suicidal ideation. ${ }^{42}$ However, the relationships between alexithymia, serum lipid levels, and suicidal ideation remain unclear.

This study was exploratory in nature and thus had several limitations that should be acknowledged. The first include the relatively small sample size and the estimation of LDL-C and VLDL-C levels by the Friedewald formula. Moreover, even if severity of OCD and suicidal ideation were analyzed using clinician-rated scales, alexithymia was assessed by a self-report scale, with possible biases due to the inherent nature of these instruments. Finally, we employed a cross-sectional design, which limits statements regarding causality, and our study lacks follow-up data. In fact, the cross-sectional nature of the present study prevents us from drawing any definite conclusions on which might be the primary phenomenon (alexithymia or dyslipidemia). Therefore, prospective studies, with repeated serum lipids evaluations before, during, and after pharmacological treatment, are needed to elucidate this. Future studies should also evaluate apolipoprotein $\mathrm{A} 1$ and $\mathrm{B} 100$, as their ratio may be a more appropriate measure than the HDL-C/LDL-C ratio.

\section{Disclosure}

The authors report no conflicts of interest.

\section{References}

1 De Berardis D, Campanella D, Serroni N, Sepede G, Carano A, Conti $\mathrm{C}$, et al. The impact of alexithymia on anxiety disorders: a review of the literature. Curr Psychiatry Rev. 2008;4:80-6.

2 De Berardis D, Campanella D, Gambi F, Sepede G, Salini G, Carano $A$, et al. Insight and alexithymia in adult outpatients with obsessivecompulsive disorder. Eur Arch Psychiatry Clin Neurosci. 2005;255:350-8.

3 De Berardis D, Conti CM, Serroni N, Moschetta FS, Carano A, Salerno RM, et al. The role of cholesterol levels in mood disorders and suicide. J Biol Regul Homeost Agents. 2009;23:133-40.

4 Balci V, Sevincok L. Suicidal ideation in patients with obsessivecompulsive disorder. Psychiatry Res. 2010;175:104-8.

5 De Berardis D, Serroni N, Campanella D, Carano A, Caltabiano M, Pizzorno AM, et al. Rischio suicidario in pazienti con disturbo ossessivo-compulsivo: il ruolo dell'alessitimia e dell'insight. Giorn Ital Psicopat. 2008;14:185-96.

6 Hintikka J, Honkalampi K, Koivumaa-Honkanen $\mathrm{H}$, Antikainen $\mathrm{R}$, Tanskanen A, Haatainen K, et al. Alexithymia and suicidal ideation: a 12-month follow-up study in a general population. Compr Psychiatry. 2004;45:340-5.

7 Pompili M. Exploring the phenomenology of suicide. Suicide Life Threat Behav. 2010;40:234-44.

8 De Berardis D, Campanella D, Serroni N, Moschetta FS, Di Emidio F, Conti $\mathrm{C}$, et al. Alexithymia, suicide risk and serum lipid levels among adult outpatients with panic disorder. Compr Psychiatry. 2013; 54:517-22.

9 Papakostas GI, Ongür D, losifescu DV, Mischoulon D, Fava M. Cholesterol in mood and anxiety disorders: review of the literature and new hypotheses. Eur Neuropsychopharmacol. 2004;14:135-42.

10 De Berardis D, Serroni N, Campanella D, Carano A, Gambi F, Valchera $A$, et al. Alexithymia and its relationships with C-reactive protein and serum lipid levels among drug naïve adult outpatients with major depression. Prog Neuropsychopharmacol Biol Psychiatry. 2008;32:1982-6.

11 Peter H, Hand I, Hohagen F, Koenig A, Mindermann O, Oeder F, et al. Serum cholesterol level comparison: control subjects, anxiety disorder patients, and obsessive-compulsive disorder patients. Can J Psychiatry. 2002;47:557-61.

12 Agargun MY, Dulger H, Inci R, Kara H, Ozer OA, Sekeroglu MR, et al. Serum lipid concentrations in obsessive-compulsive disorder patients with and without panic attacks. Can J Psychiatry. 2004; 49:776-8.

13 Fiedorowicz JG, Palagummi NM, Behrendtsen O, Coryell WH. Cholesterol and affective morbidity. Psychiatry Res. 2010;175:78-81.

14 Papadopoulou A, Markianos M, Christodoulou C, Lykouras L. Plasma total cholesterol in psychiatric patients after a suicide attempt and in follow-up. J Affect Disord. 2013;148:440-3.

15 Perez-Rodriguez MM, Baca-Garcia E, Diaz-Sastre C, Garcia-Resa E, Ceverino A, Saiz-Ruiz J, et al. Low serum cholesterol may be associated with suicide attempt history. J Clin Psychiatry. 2008;69:1920-7.

16 Goodman WK, Price LH, Rasmussen SA, Mazure C, Fleischmann $\mathrm{RL}$, Hill CL, et al. The Yale-Brown Obsessive Compulsive Scale. I. Development, use, and reliability. Arch Gen Psychiatry. 1989; 46:1006-11.

17 Mataix-Cols D, Rauch SL, Manzo PA, Jenike MA, Baer L. Use of factor-analyzed symptom dimensions to predict outcome with serotonin reuptake inhibitors and placebo in the treatment of obsessive-compulsive disorder. Am J Psychiatry. 1999;156:1409-16.

18 Bressi C, Taylor G, Parker J, Bressi S, Brambilla V, Aguglia E, et al. Cross validation of the factor structure of the 20 -item Toronto 
Alexithymia Scale: an Italian multicenter study. J Psychosom Res. 1996;41:551-9.

19 Beck AT, Kovacs M, Weissman A. Assessment of suicidal intention: the Scale for Suicide Ideation. J Consult Clin Psychol. 1979;47:343-52.

20 Montgomery SA, Asberg M. A new depression scale designed to be sensitive to change. Br J Psychiatry. 1979;134:382-9.

21 Freyberger $\mathrm{H}$. Supportive psychotherapeutic techniques in primary and secondary alexithymia. Psychother Psychosom. 1977;28:33742.

22 Zhang J, McKeown RE, Hussey JR, Thompson SJ, Woods JR, Ainsworth BE. Low HDL cholesterol is associated with suicide attempt among young healthy women: the Third National Health and Nutrition Examination Survey. J Affect Disord. 2005;89:25-33.

23 lancu I, Dannon PN, Poreh A, Lepkifker E, Grunhaus L. Alexithymia and suicidality in panic disorder. Compr Psychiatry. 2001;42:477-81.

24 Marasco V, De Berardis D, Serroni N, Campanella D, Acciavatti T, Caltabiano $\mathrm{M}$, et al. [Alexithymia and suicide risk among patients with schizophrenia: preliminary findings of a cross-sectional study]. Riv Psichiatr. 2011;46:31-7.

25 Kauhanen J, Kaplan GA, Cohen RD, Julkunen J, Salonen JT. Alexithymia and risk of death in middle-aged men. J Psychosom Res. 1996;41:541-9.

26 Sakuraba S, Kubo M, Komoda T, Yamana J. Suicidal ideation and alexithymia in patients with alcoholism: a pilot study. Subst Use Misuse. 2005;40:823-30.

27 Torres AR, de Abreu Ramos-Cerqueira AT, Torresan RC, de Souza Domingues $M$, Hercos $A C$, Guimarães $A B$. Prevalence and associated factors for suicidal ideation and behaviors in obsessivecompulsive disorder. CNS Spectr. 2007;12:771-8.

28 Apter A, Horesh N, Gothelf D, Zalsman G, Erlich Z, Soreni N, et al. Depression and suicidal behavior in adolescent inpatients with obsessive compulsive disorder. J Affect Disord. 2003;75:181-9.

29 Pfrieger FW. Cholesterol homeostasis and function in neurons of the central nervous system. Cell Mol Life Sci. 2003;60:1158-71.

30 Lalovic A, Levy E, Luheshi G, Canetti L, Grenier E, Sequeira A, et al. Cholesterol content in brains of suicide completers. Int $\mathrm{J}$ Neuropsychopharmacol. 2007;10:159-66.

31 Freemantle E, Mechawar N, Turecki G. Cholesterol and phospholipids in frontal cortex and synaptosomes of suicide completers:
Relationship with endosomal lipid trafficking genes. J Psychiatr Res. 2013;47:272-9.

32 Vevera J, Fisar Z, Kvasnicka T, Zdenek H, Stárková L, Ceska R, et al. Cholesterol-lowering therapy evokes time-limited changes in serotonergic transmission. Psychiatry Res. 2005;133:197-203.

33 Wallner B, Machatschke IH. The evolution of violence in men: the function of central cholesterol and serotonin. Prog Neuropsychopharmacol Biol Psychiatry. 2009;33:391-7.

34 Ruljancic N, Mihanovic M, Cepelak I. Thrombocyte serotonin and serum cholesterol concentration in suicidal and non-suicidal depressed patients. Prog Neuropsychopharmacol Biol Psychiatry. 2011;35:1261-7.

35 Zhang J. Epidemiological link between low cholesterol and suicidality: a puzzle never finished. Nutr Neurosci. 2011;14:268-87.

36 Kuloglu M, Atmaca M, Tezcan E, Gecici O, Tunckol H, Ustundag B. Antioxidant enzyme activities and malondialdehyde levels in patients with obsessive-compulsive disorder. Neuropsychobiology. 2002; 46:27-32.

37 Behl A, Swami G, Sircar SS, Bhatia MS, Banerjee BD. Relationship of possible stress-related biochemical markers to oxidative/antioxidative status in obsessive-compulsive disorder. Neuropsychobiology 2010;61:210-4.

38 Richter J, Möller B, Spitzer C, Letzel S, Bartols S, Barnow S, et al. Transcallosal inhibition in patients with and without alexithymia. Neuropsychobiology. 2006;53:101-7.

39 Kano M, Fukudo S, Gyoba J, Kamachi M, Tagawa M, Mochizuki H, et al. Specific brain processing of facial expressions in people with alexithymia: an H2 15O-PET study. Brain. 2003;126:1474-84.

40 Berthoz S, Artiges E, Van De Moortele PF, Poline JB, Rouquette S, Consoli SM, et al. Effect of impaired recognition and expression of emotions on frontocingulate cortices: an fMRI study of men with alexithymia. Am J Psychiatry. 2002;159:961-7.

41 Del Casale A, Kotzalidis GD, Rapinesi C, Serata D, Ambrosi E, Simonetti A, et al. Functional neuroimaging in obsessive-compulsive disorder. Neuropsychobiology. 2011;64:61-85.

42 Raust A, Slama F, Mathieu F, Roy I, Chenu A, Koncke D, et al. Prefrontal cortex dysfunction in patients with suicidal behavior. Psychol Med. 2007;37:411-9. 